\title{
The Silicon Inner Tracker for LHCb
}

\author{
Olaf Steinkamp
}

- Requirements and Layout

- Silicon Sensors and Ladders

- Read-Out Chip

- Outlook

Physik Institut der Universität Zürich Winterthurerstrasse $190 \quad$ CH-8057 Zürich olaf.steinkamp@physik.unizh.ch 


\section{LHCb Experiment}

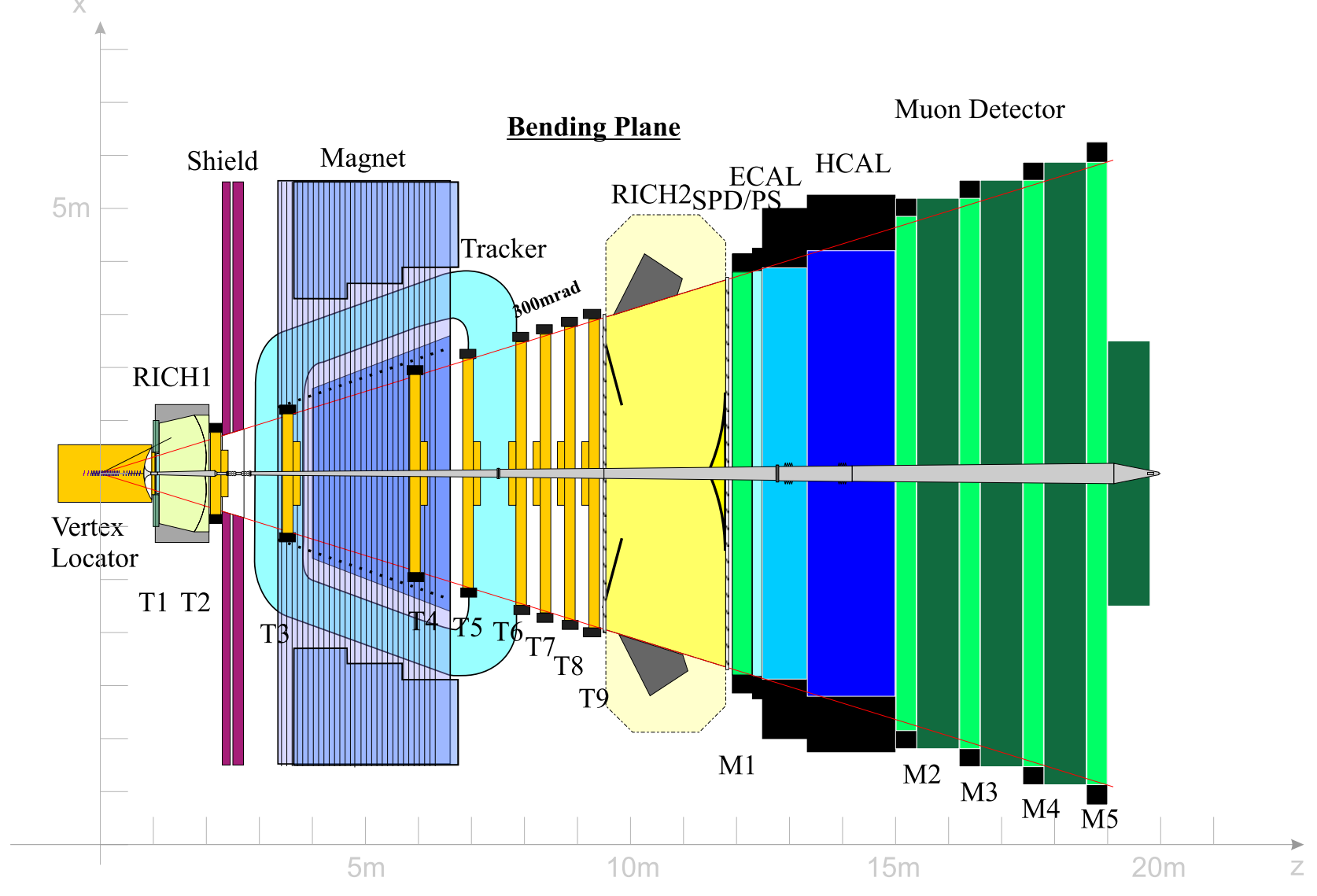

Dedicated experiment for B-physics at LHC

CP Violation and rare decays

$\mathrm{b} \overline{\mathrm{b}}$ production at LHC strongly forward peaked

$\Rightarrow$ forward spectrometer $250 \mathrm{mrad} \times 300 \mathrm{mrad}$

\section{Special attention on:}

- vertexing: VELO

$(\rightarrow$ talk by J.Libby $)$

- particle identification: RICH detectors $\quad(\rightarrow$ talk by C.Matteuzzi $)$

- triggering: VELO, calorimeters, muon detector

- tracking, momentum reconstruction: dipole spectrometer 


\section{LHCb Spectrometer}

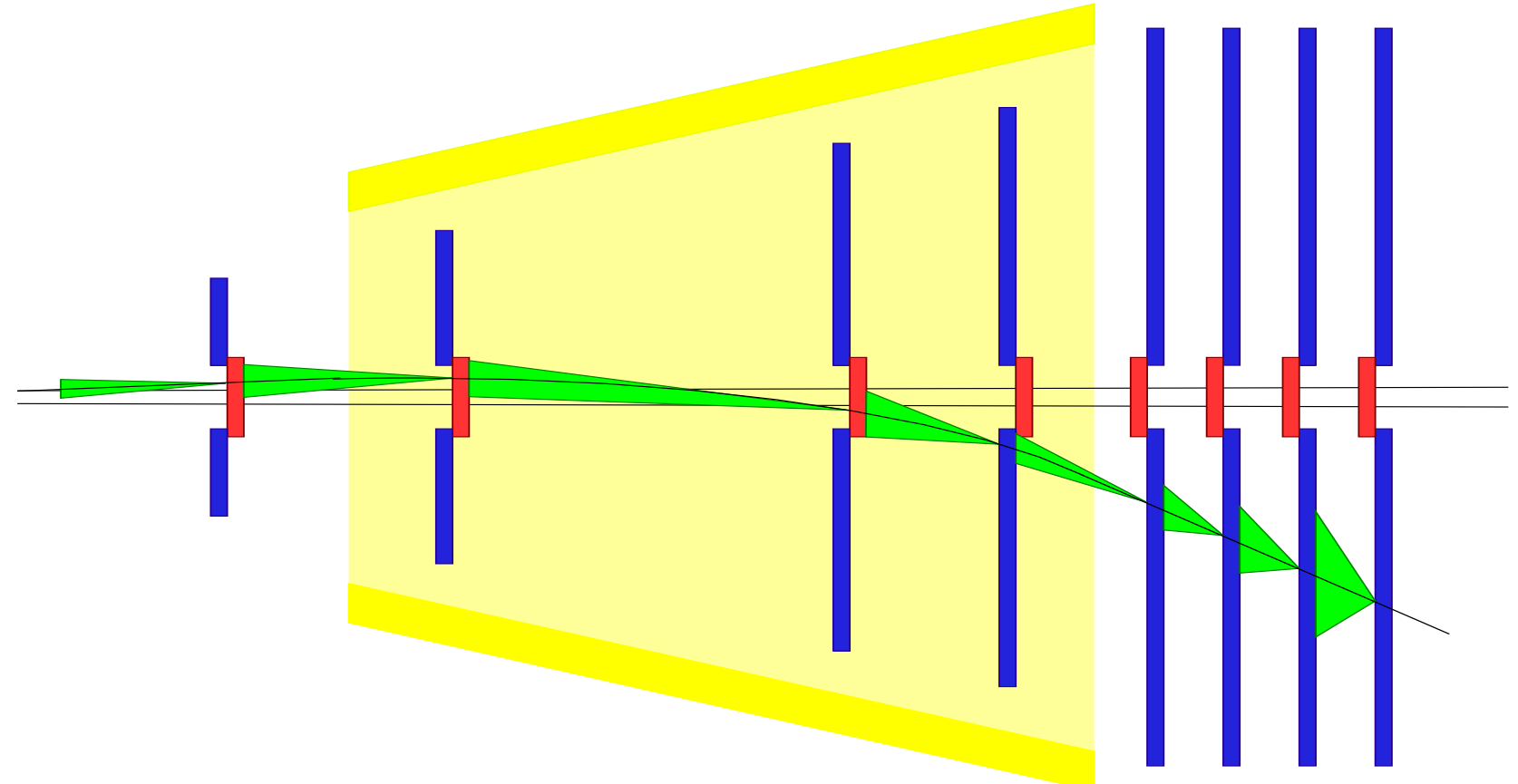

$4 \mathrm{Tm}$ dipole magnet ( bending plane horizontal)

Nine planar tracking stations

- positions optimized for "upstream" tracking

Each station four detection layers

- wire/strip orientation vertical, $+5^{\circ},-5^{\circ}$, vertical

- optimized for momentum resolution, pattern recognition

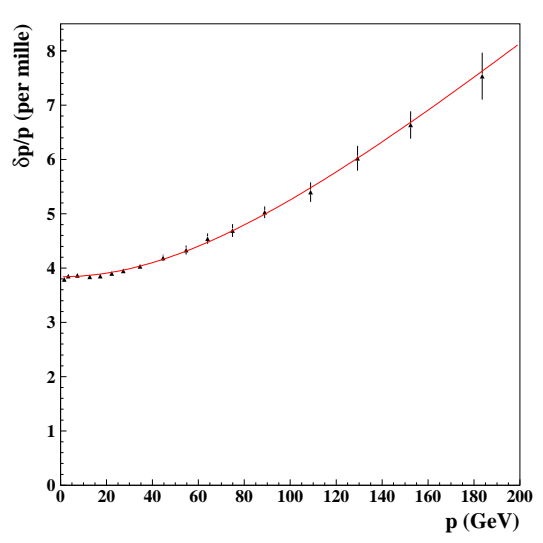

- track reconstruction efficiency $>95 \%$ for B decay tracks

- average momentum resolution $\approx 0.4 \%$, dominated by mult.scatt. up to $100 \mathrm{GeV}$

$\Rightarrow$ watch material budget! 


\section{Tracking Stations}

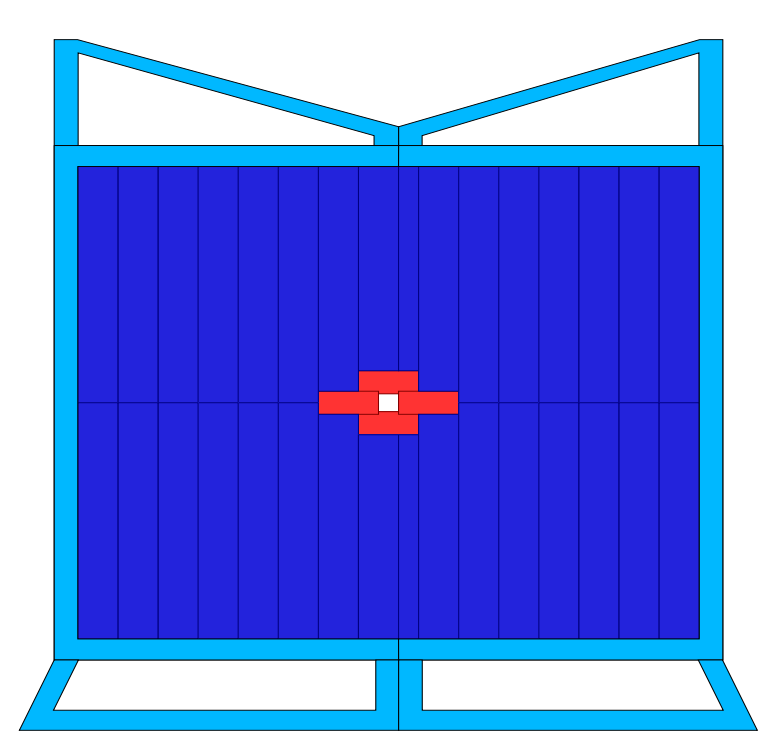

( largest station $6 \mathrm{~m} \times 5 \mathrm{~m}$ )
Track density strongly forward peaked

- up to $10^{6}$ charged part./ $\mathrm{cm}^{2}$ near beam pipe

- falling off with $\approx 1 / r$

$\Rightarrow$ each station: two detector technologies

- borderline determined by track density

- "Outer Tracker": straw drift chambers

- "Inner Tracker": silicon-strip detector

Inner Tracker: cross-shaped layout

- four detector boxes

- each box four detection layers

- each layer seven silicon ladders

- each ladder two 6" silicon sensors

- total surface 9 stations $\approx 14 \mathrm{~m}^{2}$

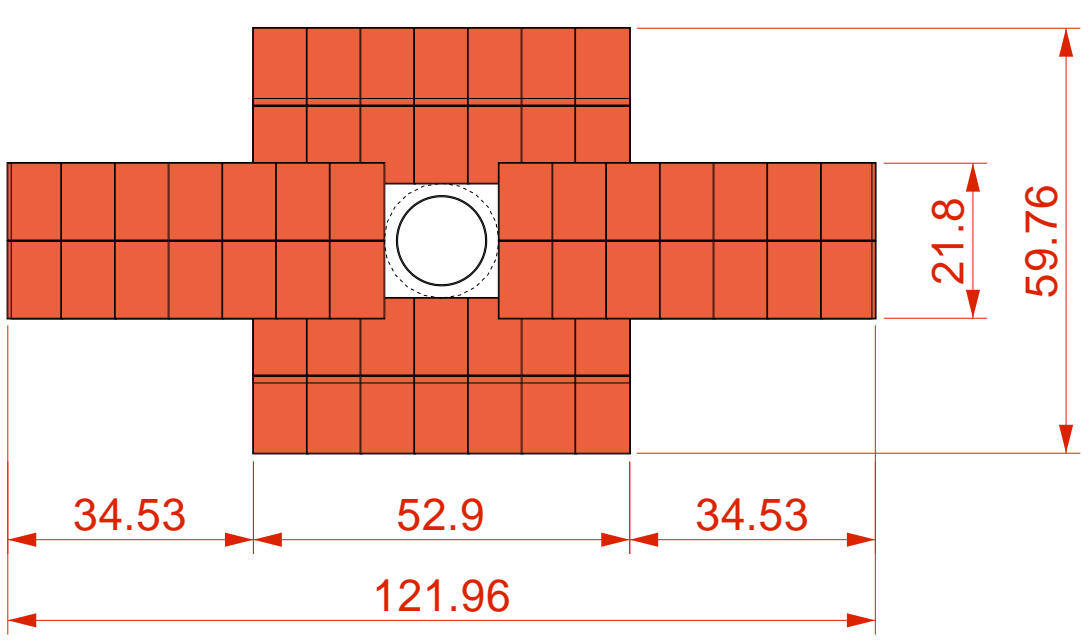




\section{Detector Simulation}

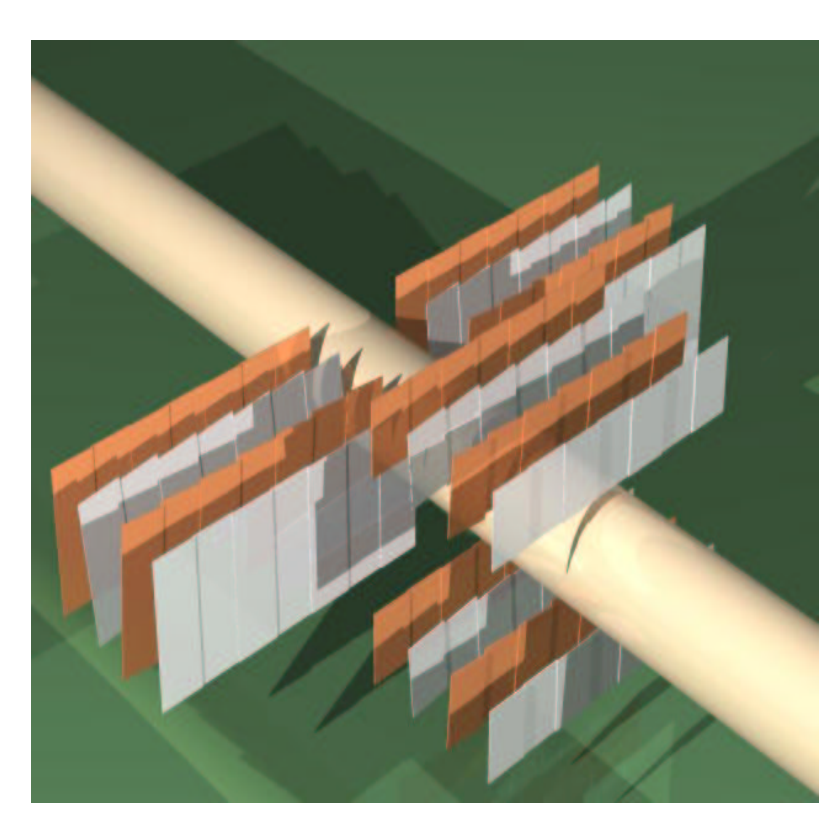

GEANT description of active and dead material Digitization within LHCb Gaudi framework:

- signal generation

- charge sharing (from laser tests)

- amplifier response (from Beetle prototype)

- gaussian noise

- clustering

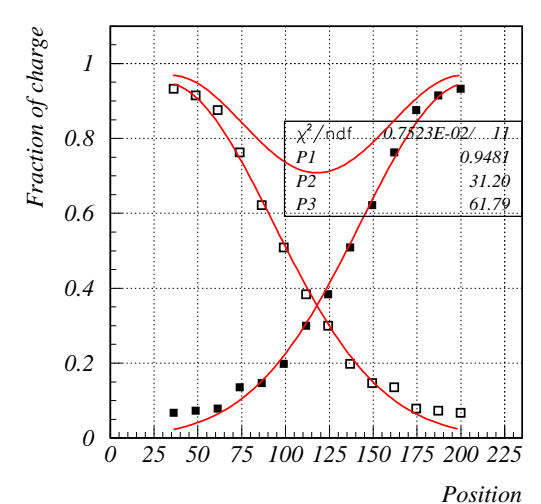

eta function

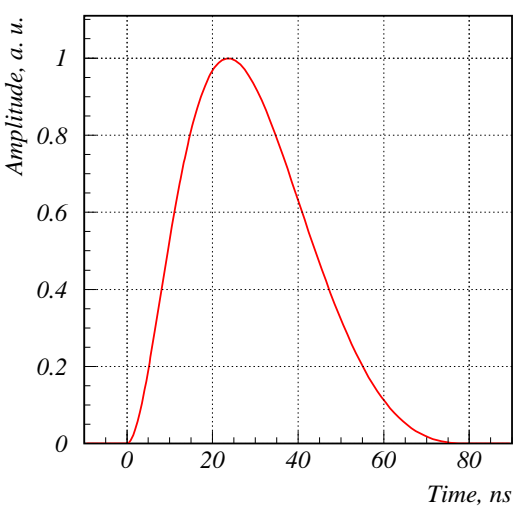

signal shape

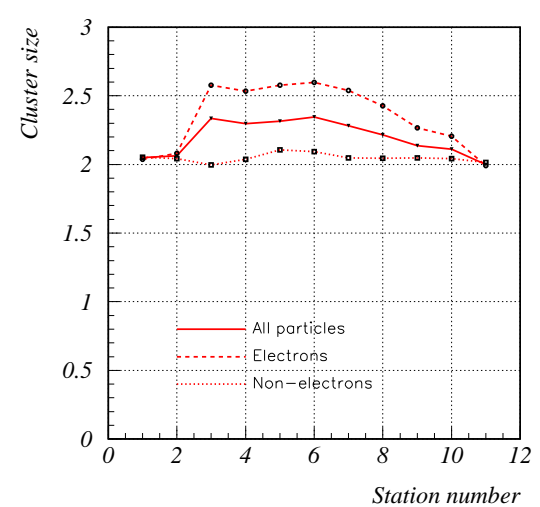

cluster size

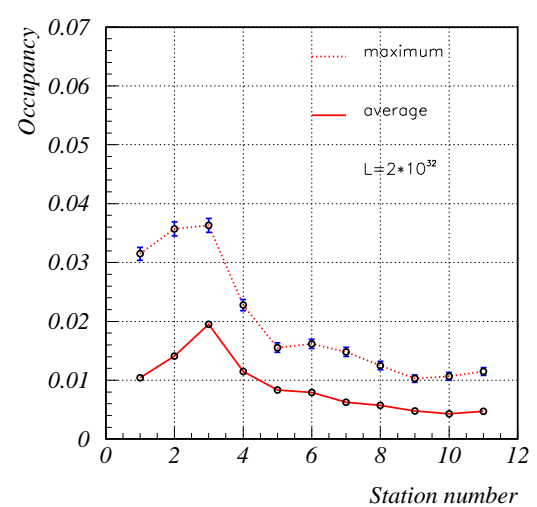

occupancies 


\section{Radiation Dose and Particle Fluxes}

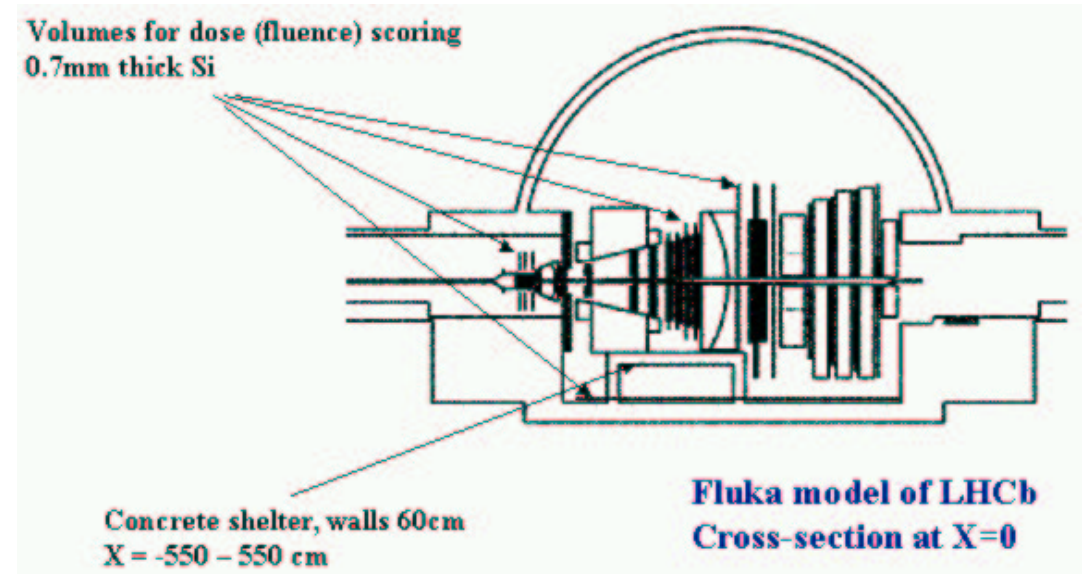

Detailed Fluka description of detector and cave

- careful analysis of Inner Tracker region to be done

From previous study:

- integrated dose $20 \mathrm{Mrad}$

- $5 \times 10^{13} 1-\mathrm{MeV}$ equiv. neutrons $/ \mathrm{cm}^{2}$

after 10 years of operation in hottest spot
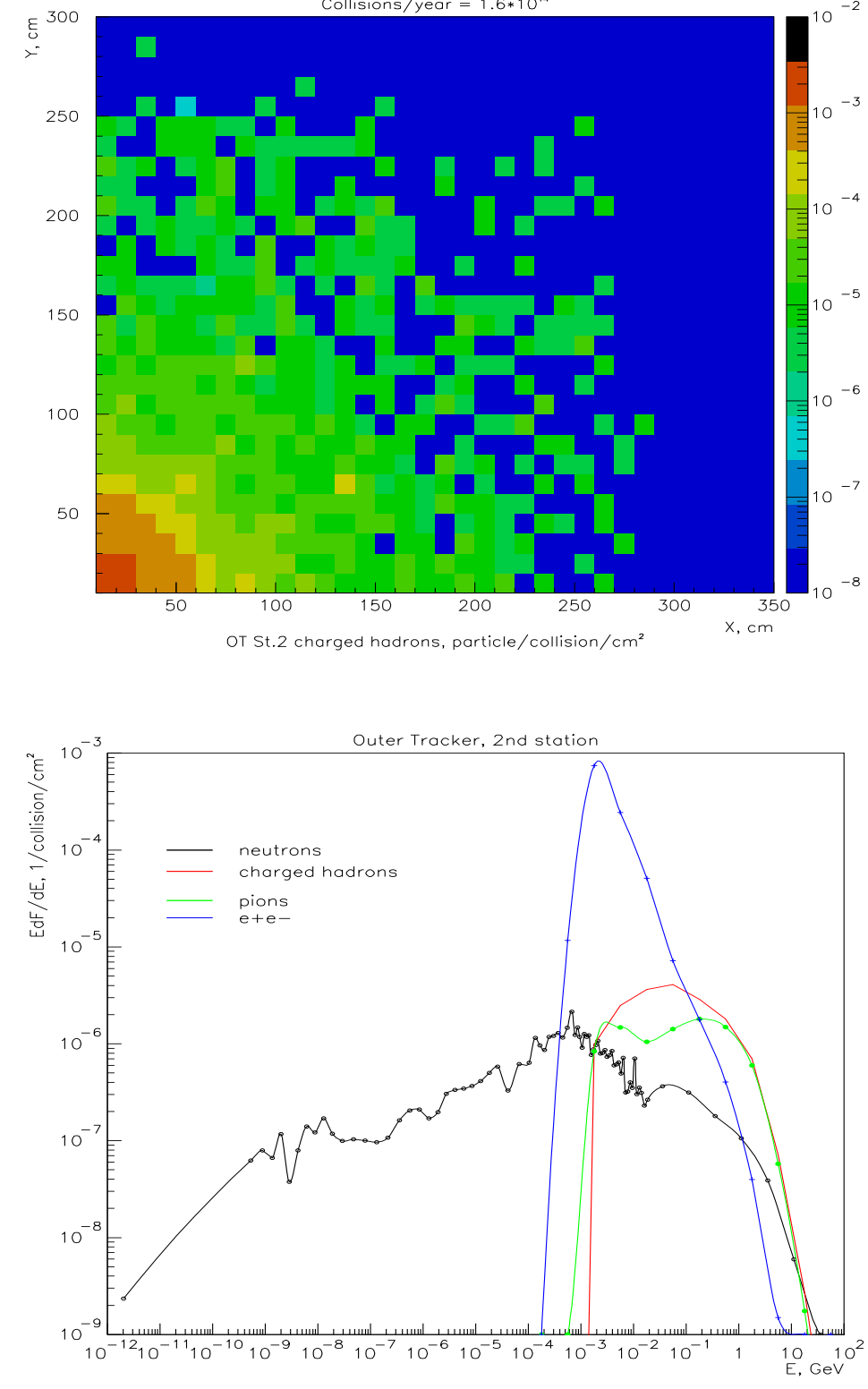


\section{Silicon Sensor Design}

Cost read-out electronics:

- "long" ladders, "large" pitch

Tracking performance, station layout:

- $22 \mathrm{~cm}$ long ladders

- readout pitch about $250 \mu \mathrm{m}$

Material budget:

- "thin" sensors

LHC bunch crossing frequency:

- shaping time $\approx 25 \mathrm{~ns}$ to avoid pile-up

- small $w / p$ to minimize capacitance

Radiation environment:

- $5 \times 10^{12} 1-\mathrm{MeV}$ equiv. $\mathrm{n} / \mathrm{cm}^{2} /$ year locally ( $\Rightarrow$ not our biggest worry ...)

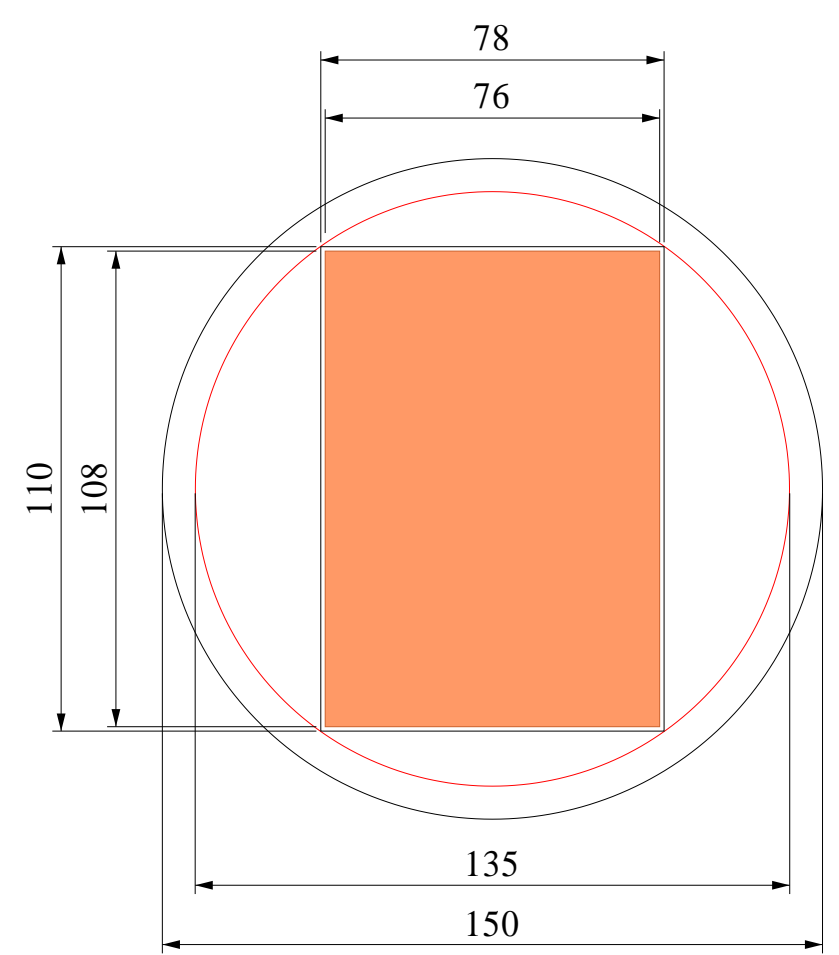

\begin{tabular}{|l||r|}
\hline Technology & $\mathrm{p}^{+}$-on-n \\
\hline Thickness & $320 \mu \mathrm{m}$ \\
\hline Sensitive & $108 \mathrm{~mm} \times 76 \mathrm{~mm}$ \\
\hline Number strips & $320(384)$ \\
\hline Strip pitch & $237.5 \mu \mathrm{m}(198 \mu \mathrm{m})$ \\
\hline Implant width & $0.2<w / p<0.3$ \\
\hline
\end{tabular}




\section{Ladder Design}

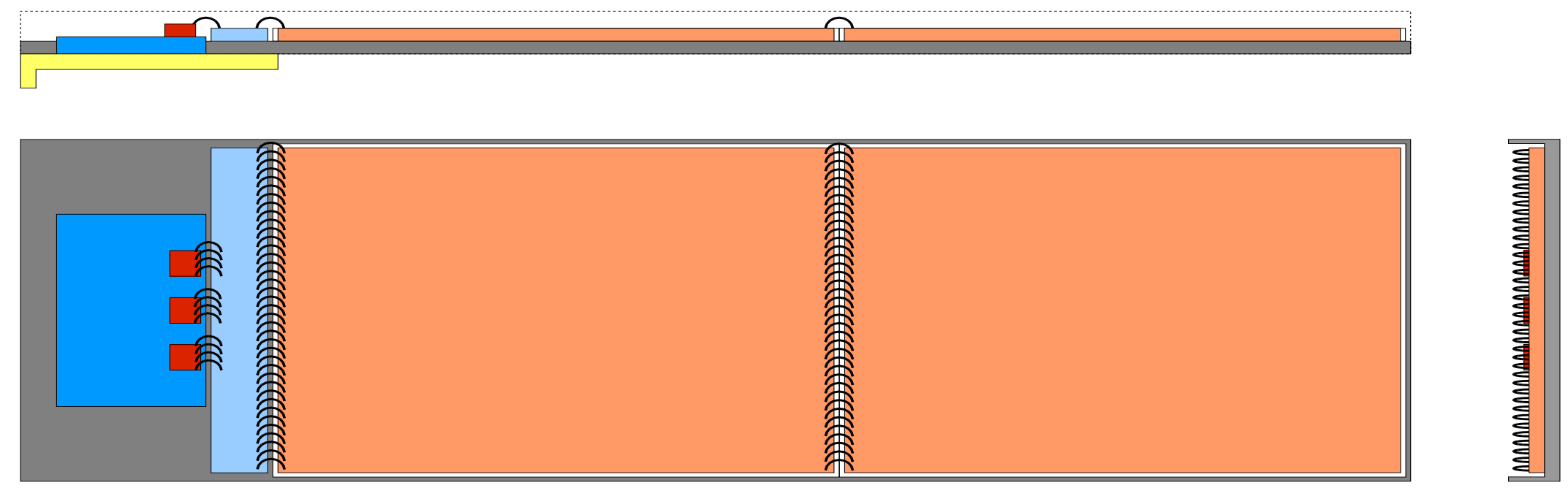

\begin{tabular}{lll}
$\square$ & Sensor & Pitch Adaptor $\square$ Readout Hybrid \\
\hline Support & Front-End Chip $\square$ Balcony
\end{tabular}

Design aims:

- simple, robust construction

- modularity

- material budget

- cooling ( sensors at $-5^{\circ} \mathrm{C}$ )

\section{Layout:}

- U-shaped carbon-fibre support

- ceramic pitch adaptor

- kapton read-out hybrid

- cooling "balcony" 


\section{Detector Box Design}

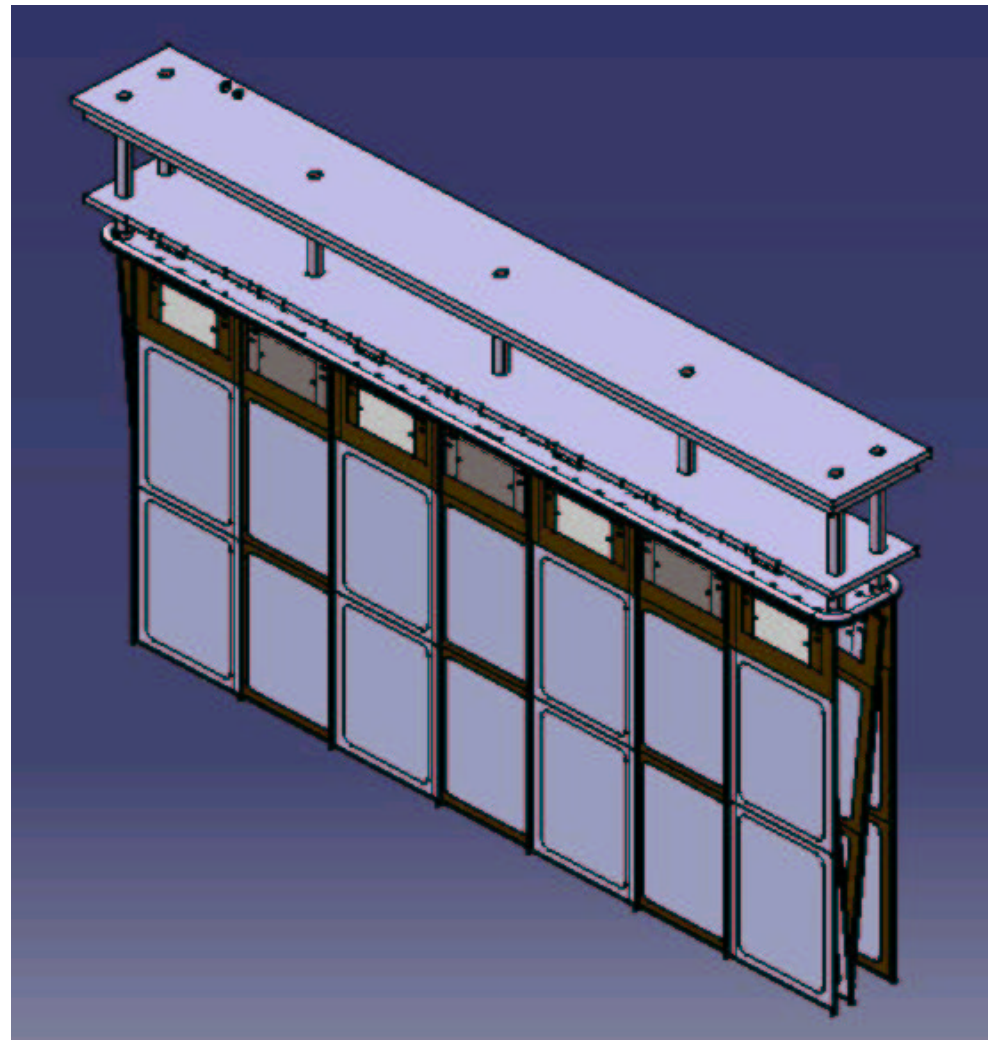

- one box for four detection layers

- common "cooling plate" carrying cooling pipe

- thermal contact and positioning via individual balconies

- cold, dry nitrogen atmosphere

- insulation box (not shown)

- light-weight foam for thermal insulation

- thin aluminium foil for electrical shielding

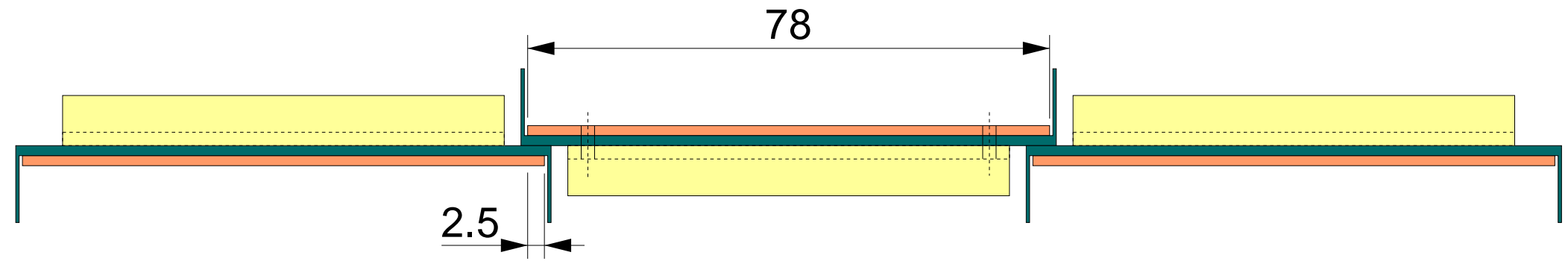




\section{Ladder Prototype}
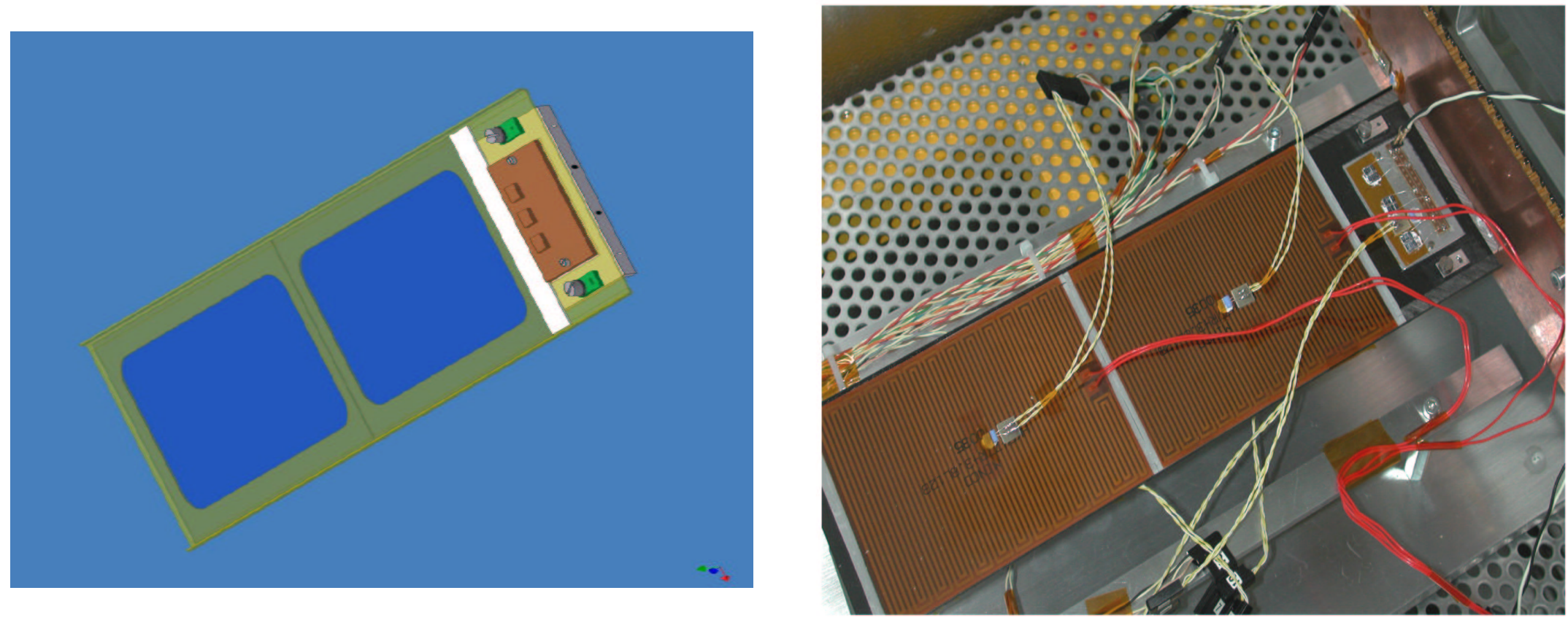

- four-layer Amoco K1100 shelf

- dummy silicon sensors and hybrid

- Kapton heat elements

- temperature probes / thermal camera

$\Rightarrow$ test mechanical and thermal properties

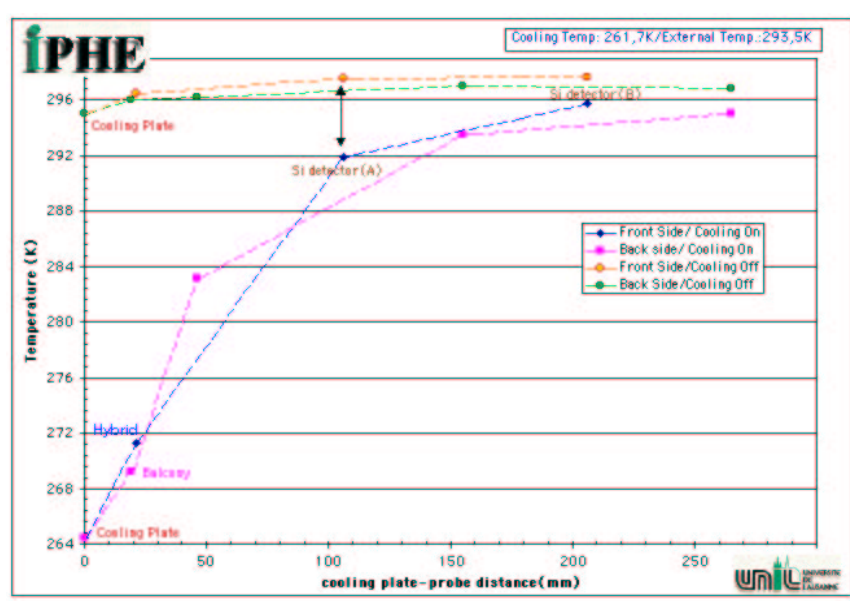




\section{Detector Box Prototype}

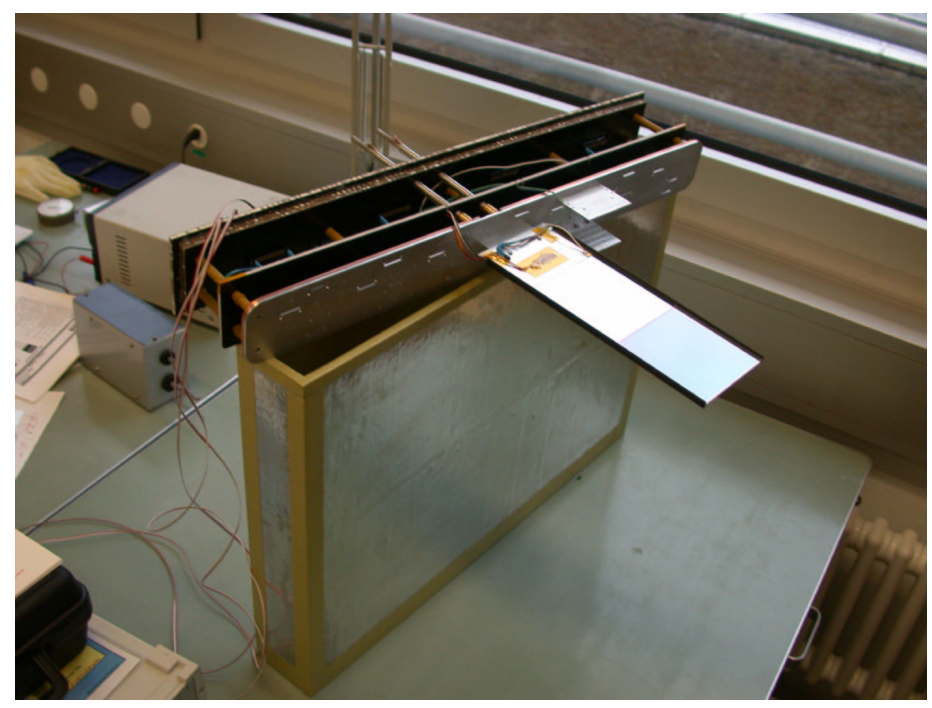

First prototype for thermal tests:

- box from mold-injected polyurethane foam

- $\mathrm{C}_{6} \mathrm{~F}_{14}$ liquid cooling

- aluminium cooling plate / balconies

- mock-up ladders with kapton heat elements

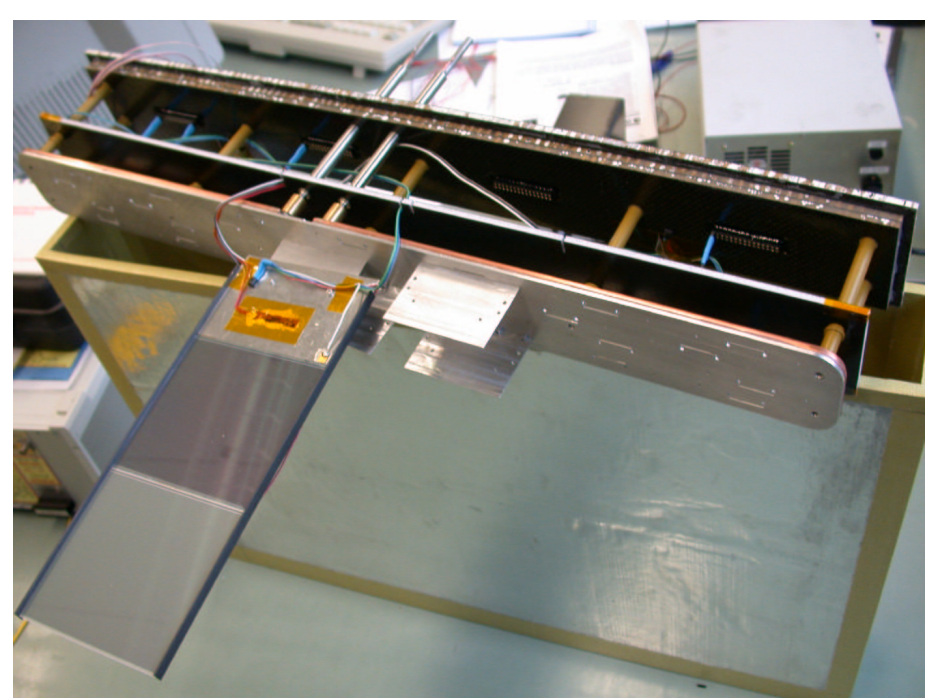

$\Rightarrow$ validate cooling concept

Next: identify low-weight materials

- balconies magnesium matrix composite (magnesium-infiltrated carbon fibres)

- cooling plate carbon-carbon material 


\section{1st Prototype Sensors from SPA-Detector, Kiev}

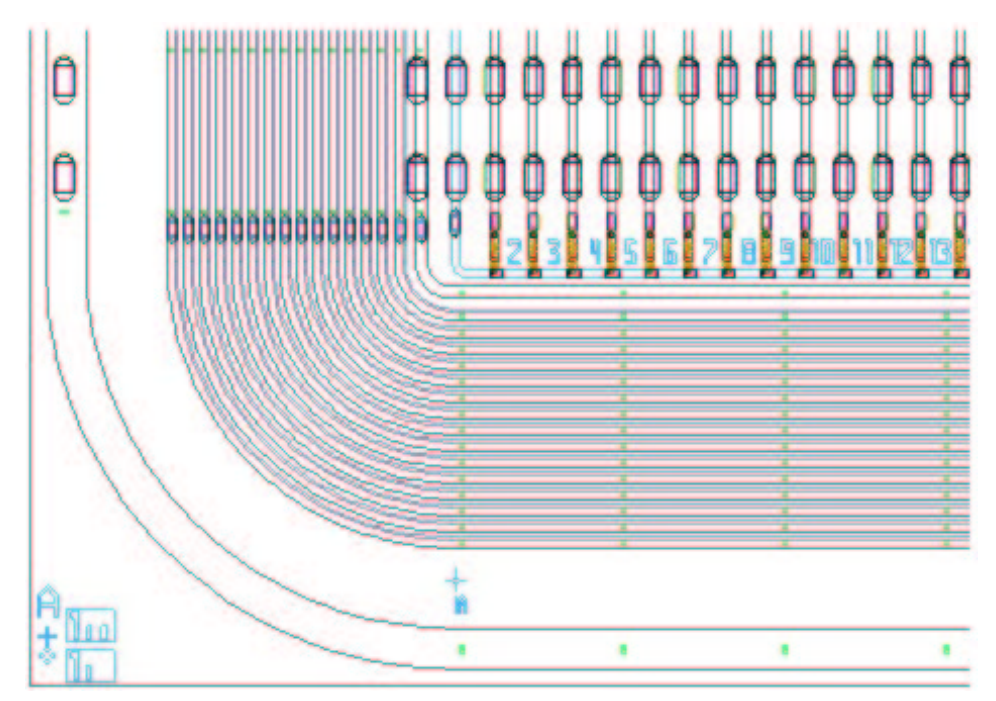

- $\mathrm{p}^{+}$-on-n, $300 \mu \mathrm{m}$ thick

- 64 strips, $6.66 \mathrm{~cm}$ long, $240 \mu \mathrm{m}$ pitch

- implant width $48,60,72 \mu \mathrm{m}$

- fast turnaround, attractive price!

- sensors work fine, except junction breakdown at too low bias voltages
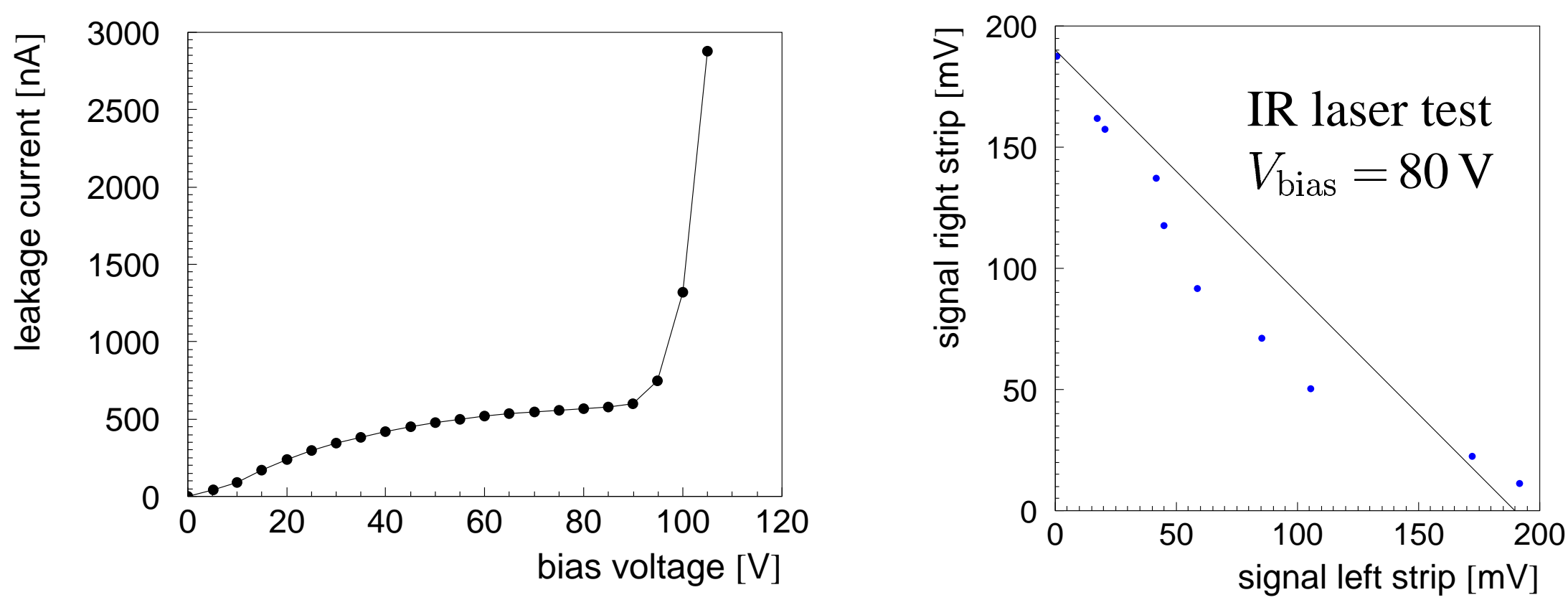


\section{1st Test Beam with SPA Prototype Sensors}

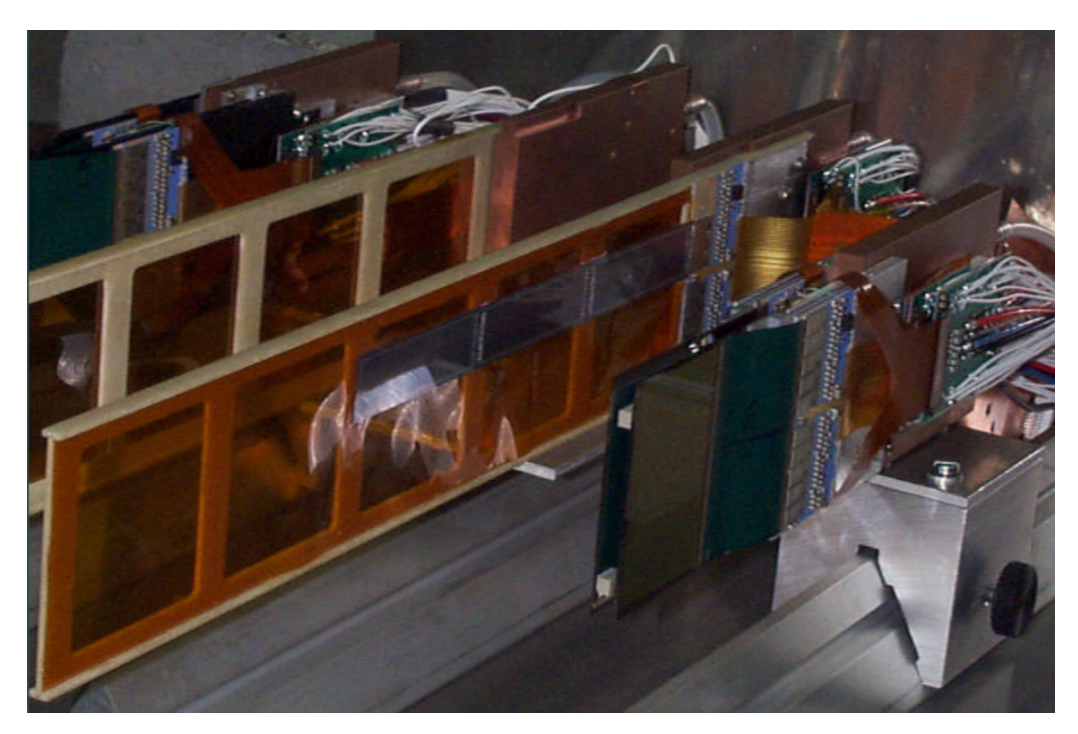

- $9 \mathrm{GeV}$ pions ( CERN-T7 test beam )

- one-sensor and three-sensor ladders

$$
\Rightarrow 6.7 \mathrm{~cm} \text { and } 20 \mathrm{~cm} \text { long }
$$

- unirradiated sensors at room temp.

- Helix read-out chip (HERA-B)

$\Rightarrow$ fastest shaping time $\approx 50 \mathrm{~ns}$
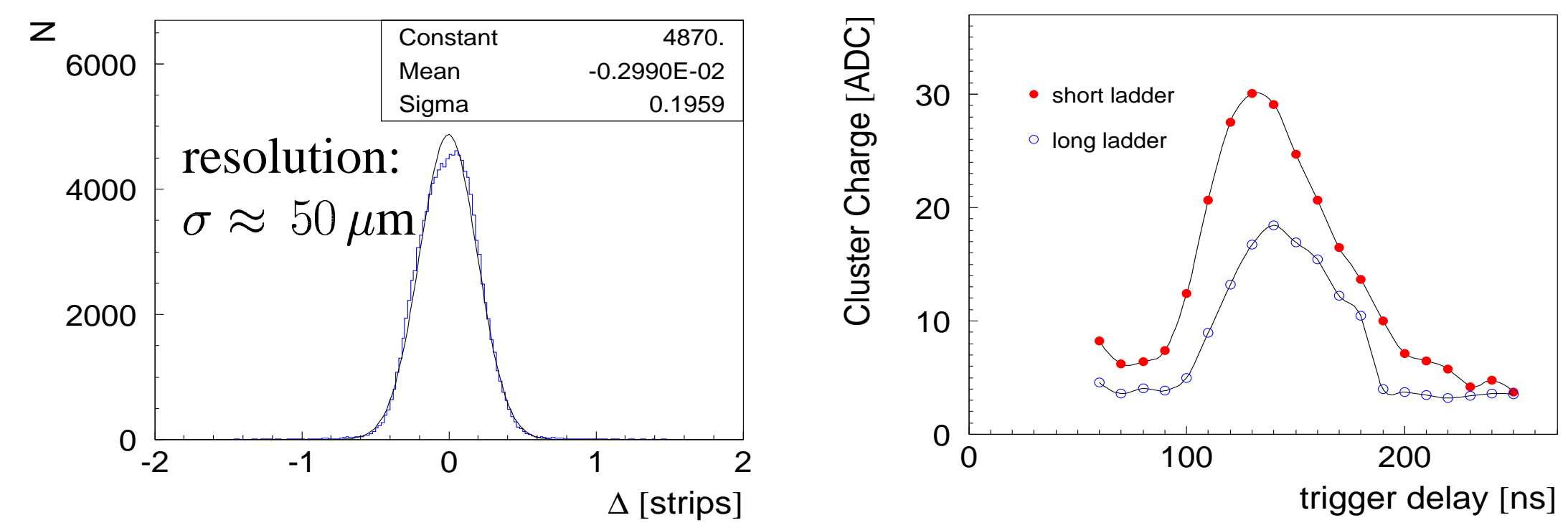


\section{Detection Efficiency 20 cm-long Ladder}

As function of $w / p\left(\right.$ for $\left.V_{\text {bias }}=90 \mathrm{~V}\right)$ :

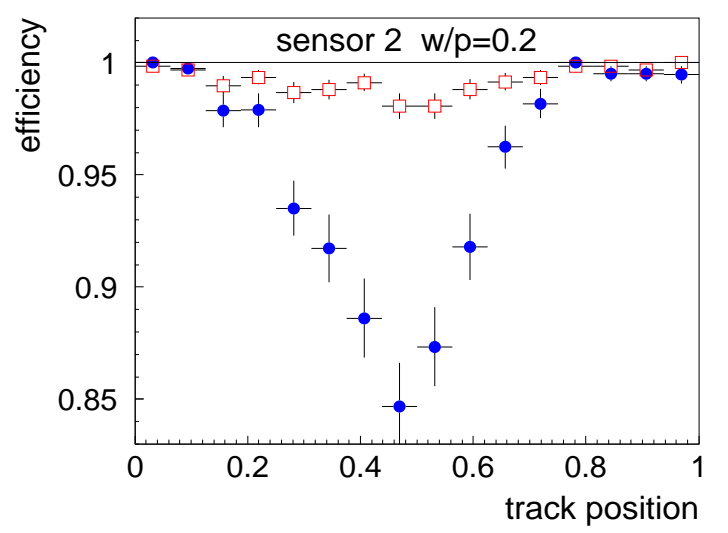

$w / p=0.2$

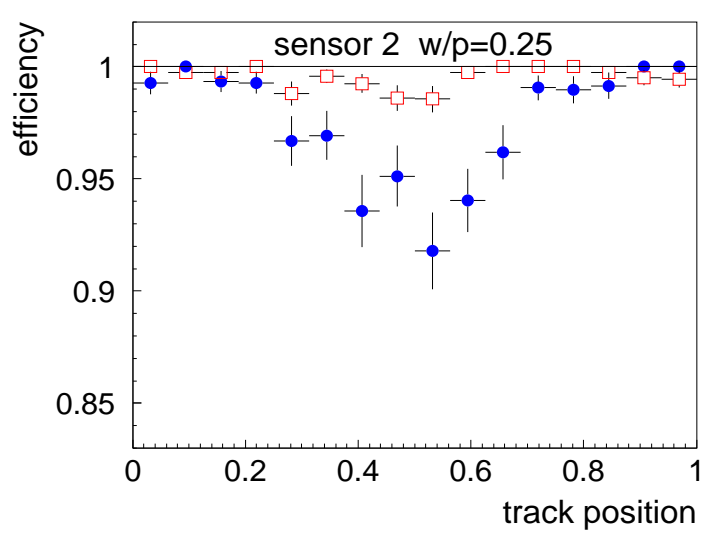

$w / p=0.25$

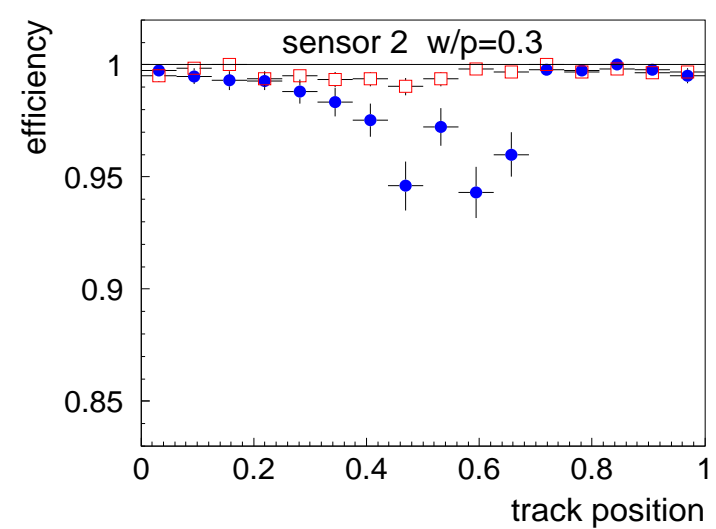

$w / p=0.3$

As function of $V_{\text {bias }}$ ( for $w / p=0.2$ ):
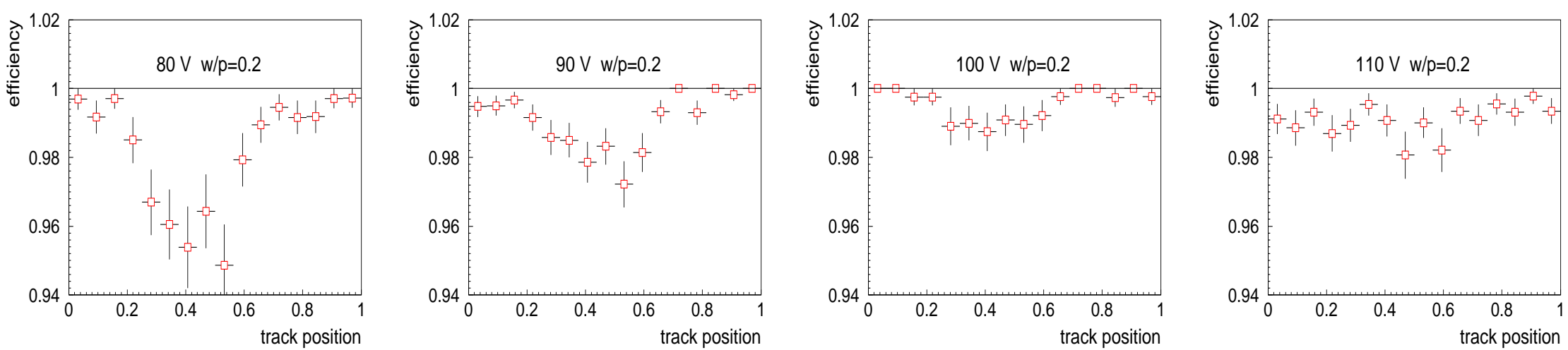

$$
V_{\text {bias }}=80 \mathrm{~V}
$$

$$
V_{\text {bias }}=90 \mathrm{~V}
$$$$
V_{\text {bias }}=100 \mathrm{~V}
$$

$V_{\text {bias }}=110 \mathrm{~V}$ 


\section{Charge Collection Efficiency}

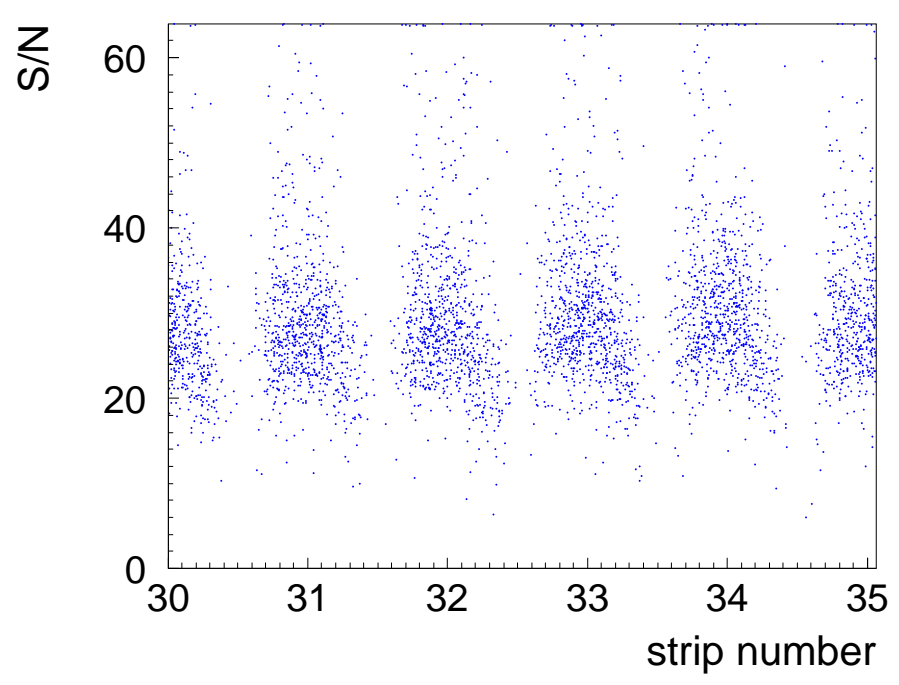

one-strip clusters

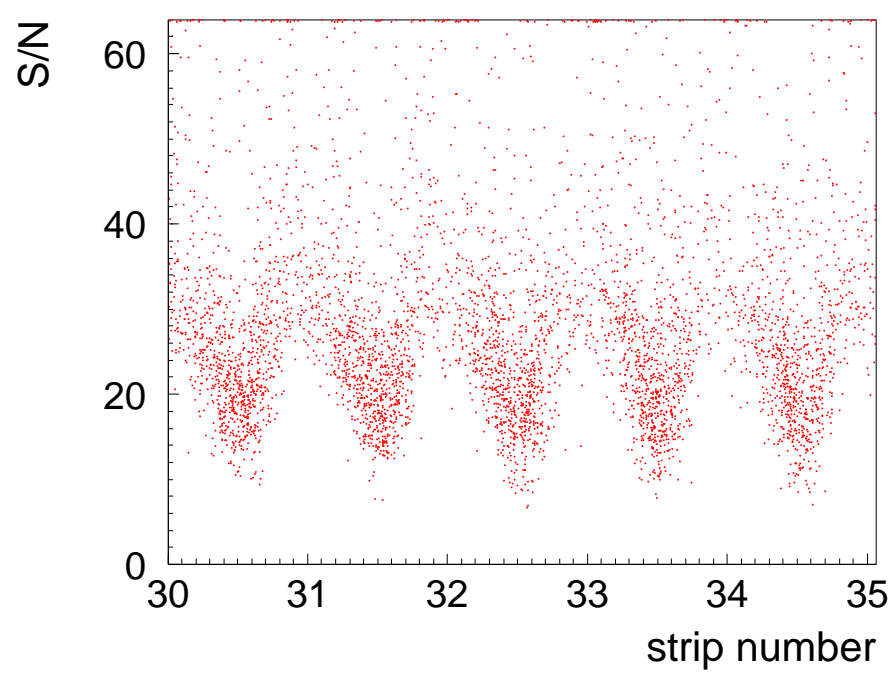

two-strip clusters

Reduced charge collection efficiency in between strips

- effect decreases with increasing $w / p$

- effect decreases with increasing overbiassing

\section{Interpretation:}

- low-field region in between strips

- charges get trapped or arrive too late ( fast shaping time!)

- ( simulation in preparation )

Cure:

- optimized strip geometry

- higher overbiassing

\section{$\Rightarrow$ new sensors with better HV performance !}




\section{Prototype Sensors from Hamamatsu}

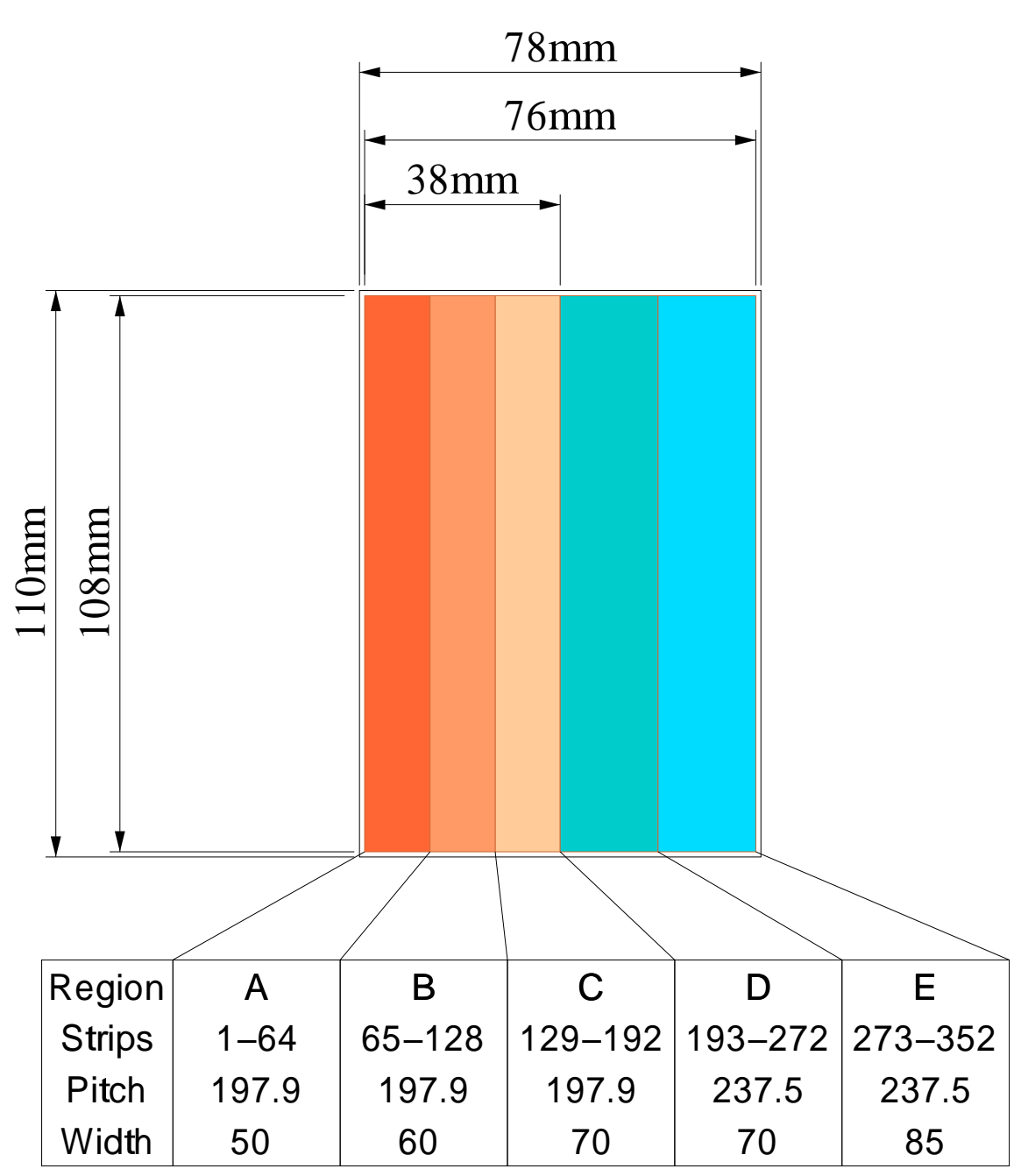

Full-size multi-geometry sensors:

- $\mathrm{p}^{+}$-on-n, $320 \mu \mathrm{m}$ thick

- $198 \mu \mathrm{m}$ and $237.5 \mu \mathrm{m}$ pitch

- $0.2<w / p<0.3$

Sensors received last Friday:

$\Rightarrow$ testbeam at CERN end May

$\Rightarrow$ select strip geometry for $\mathrm{LHCb}$

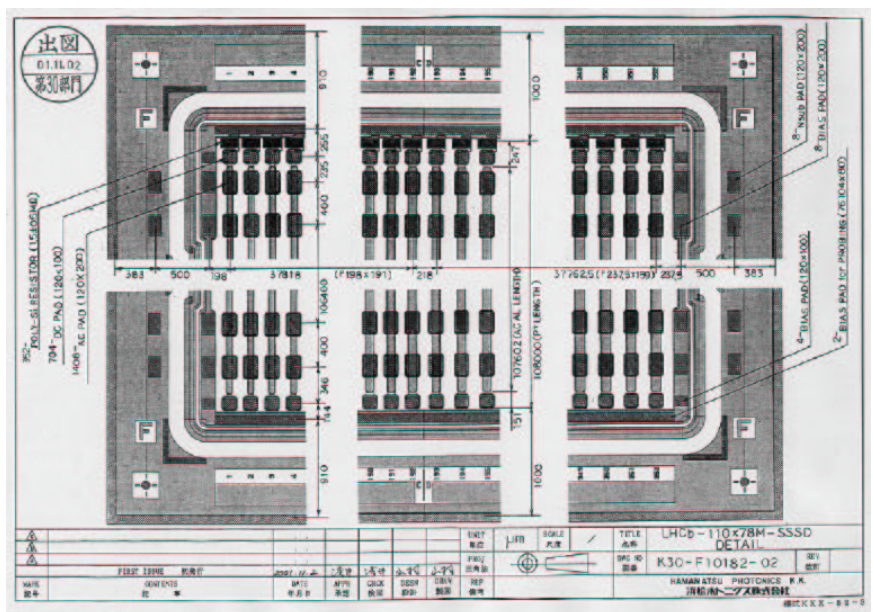




\section{Beetle Readout Chip}

128-channel pipelined r/o chip, custom design at ASIC lab Heidelberg

- basic RD20 front-end architecture

- $0.25 \mu \mathrm{m}$ CMOS

- analog and binary pipeline

- prompt binary readout

- programmable via $\mathrm{I}^{2} \mathrm{C}$ interface

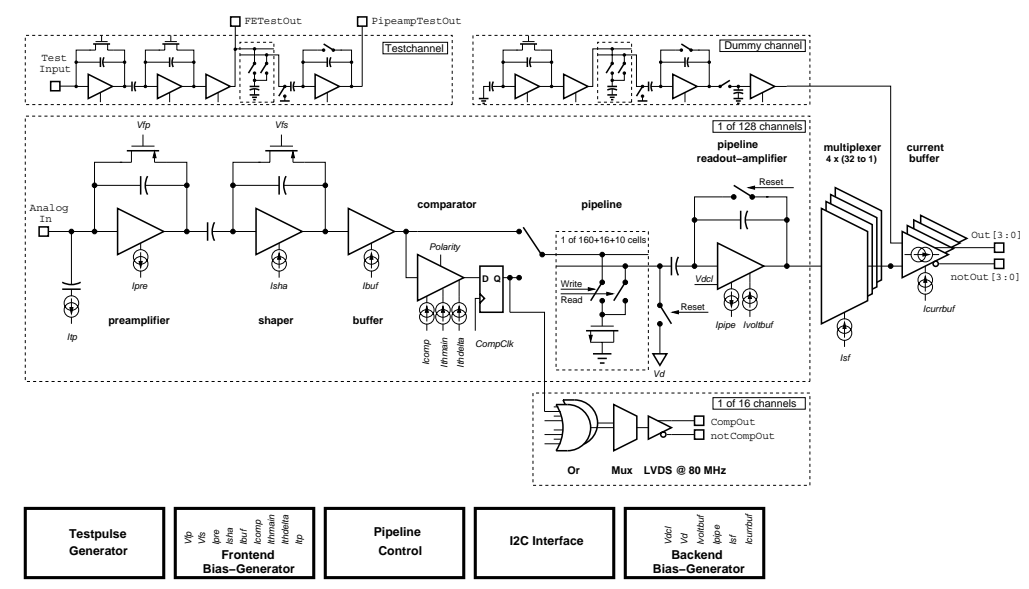

$\mathrm{LHCb}$ front-end requirements

- $40 \mathrm{MHz}$ sampling rate

- $1 \mathrm{MHz}$ read-out rate

- 160 cell analog pipeline

- 16 event internal buffer

- 900 ns max. read-out time

Beetle 1.1 submitted March 2001

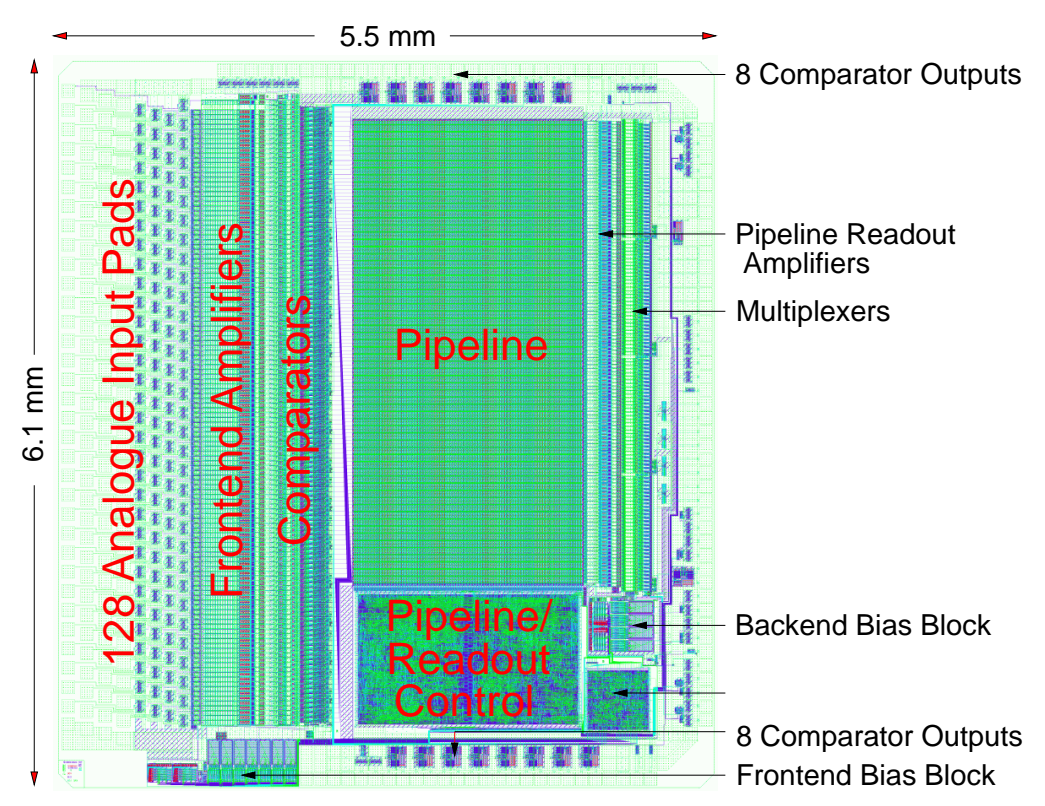




\section{Beetle 1.1 Total Ionizing Dose Irradiation}

Four chips irradiated at CERN X-ray facility

$\Rightarrow$ full functionality up to $45 \mathrm{Mrad}$

trigger / readout and slow control

$\Rightarrow$ performance degradation small

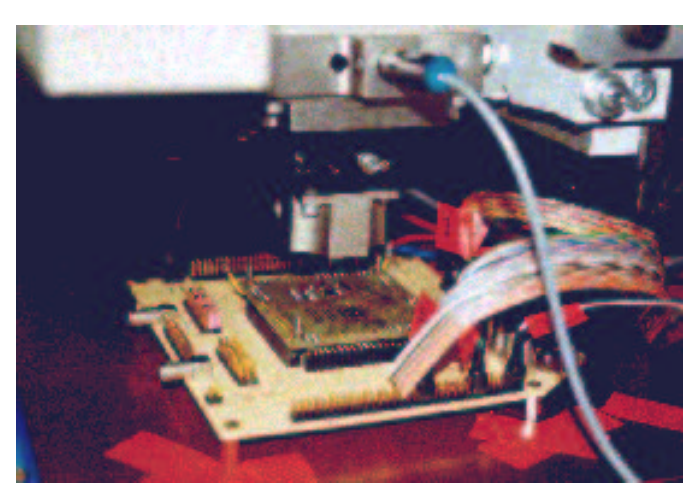

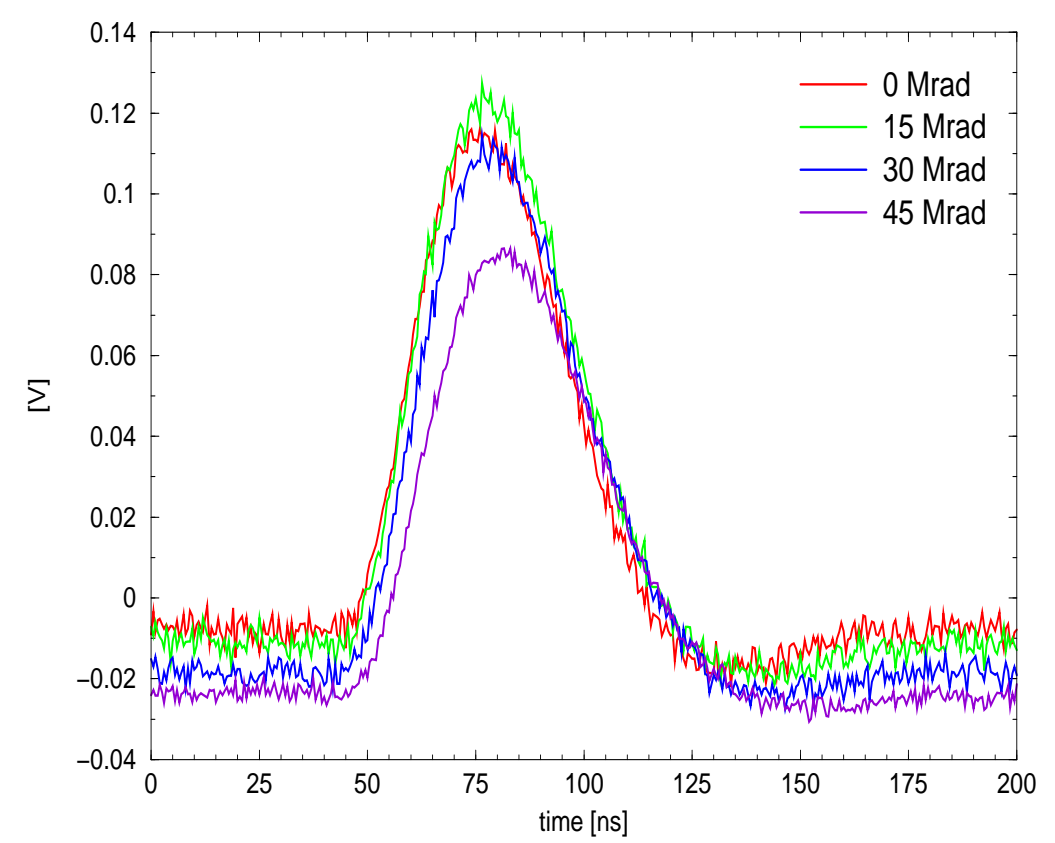

Pulse shape at $\mathrm{C}_{\text {load }}=3 \mathrm{pF}$

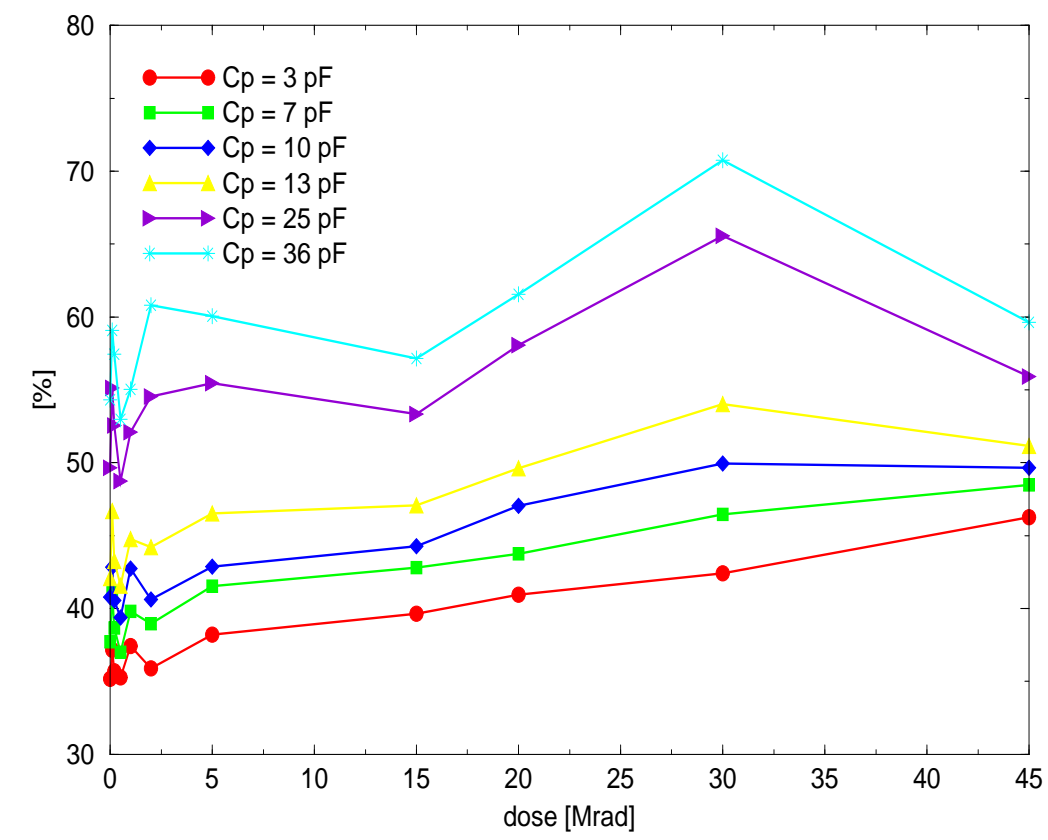

Amplitude remainder after $25 \mathrm{~ns}$ 


\title{
1st Test Beam with Beetle 1.1 Read-Out Chip
}

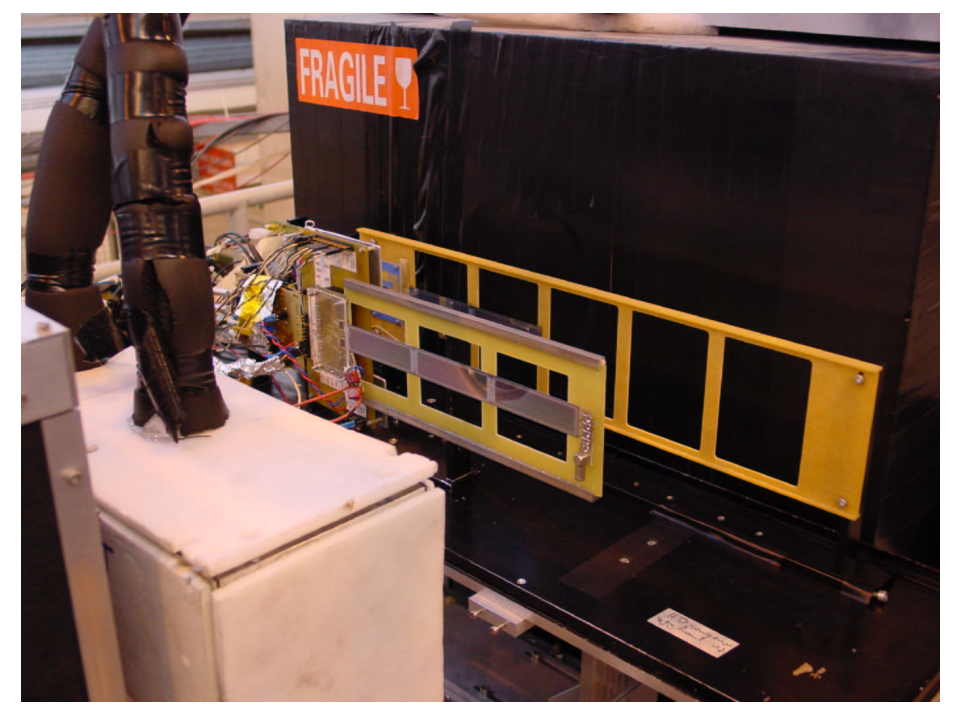

- $120 \mathrm{GeV}$ muons (CERN-X7 test beam )

- SPA prototype sensor ladders

\author{
$6.7 \mathrm{~cm}$ and $20 \mathrm{~cm}$ long \\ $\Rightarrow$ successful, reliable operation of chips
}

\section{( S / N limited by DAQ setup )}
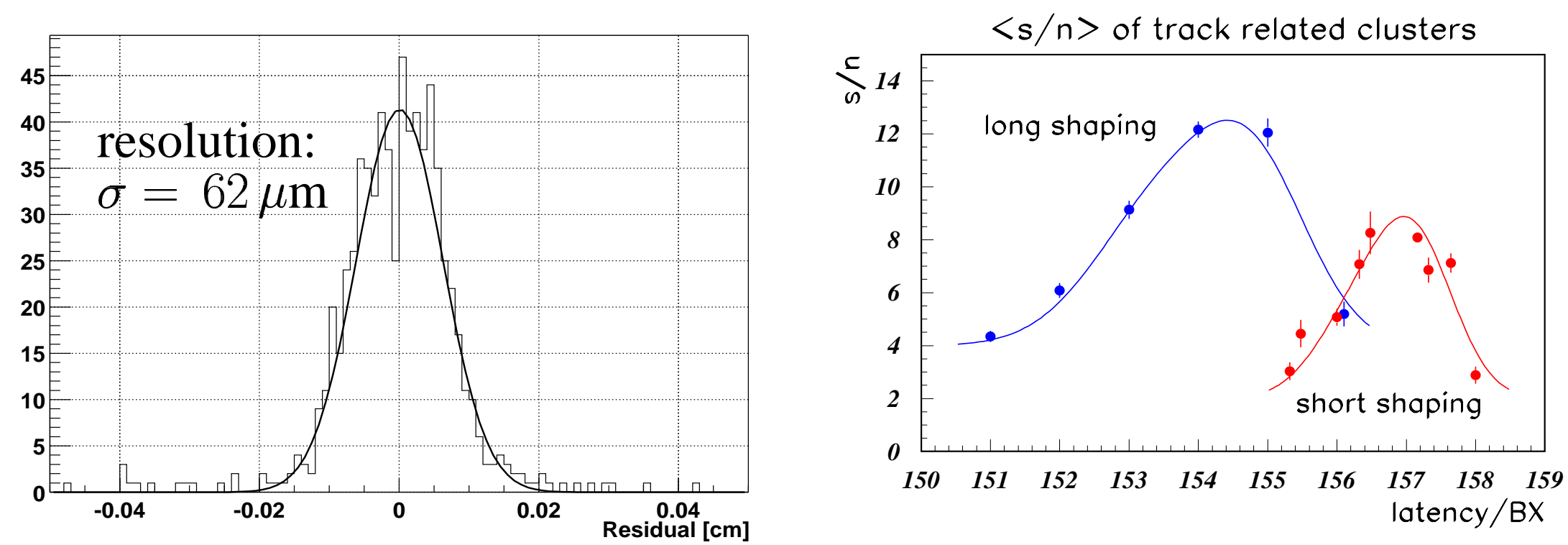


\section{Beetle — Next Steps}

Beetle 1.2, submission April 2002:

- few bug fixes

- SEU redundant logic

- fully differential output driver

- improved front-ends

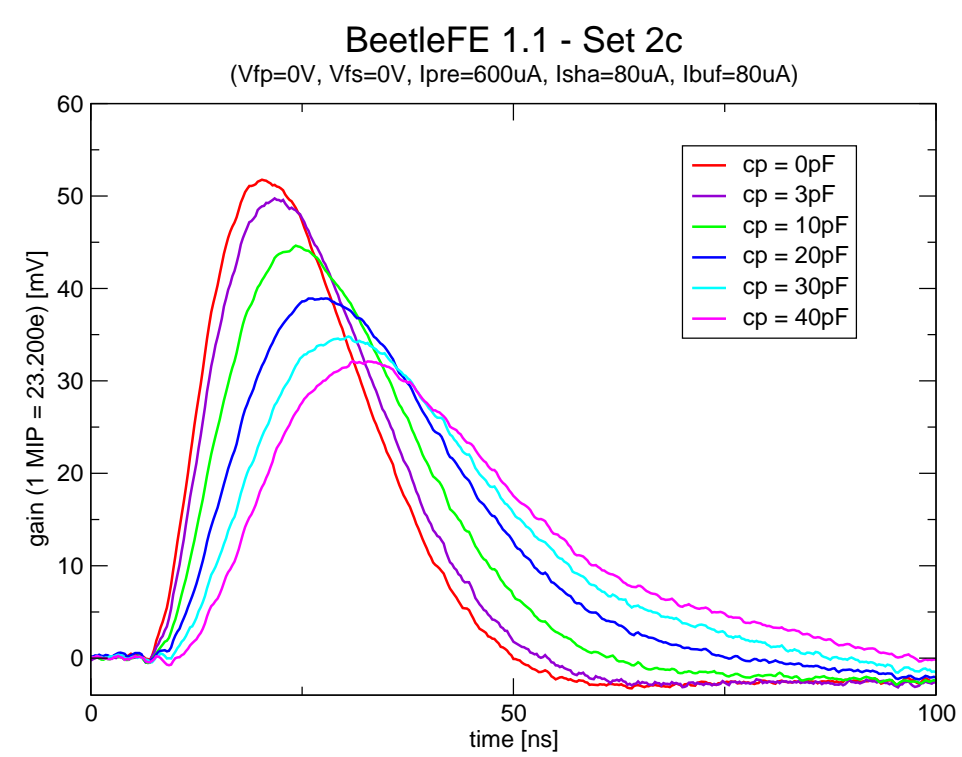

Measured on test chips (Beetle-FE 1.1):

- remainder after $25 \mathrm{~ns}$

$$
<30 \% \text { for } C_{\text {load }} \text { up to } 30 \mathrm{pF}
$$

- ENC

$$
\begin{aligned}
& \approx 520 \mathrm{e}^{-}+40 \mathrm{e}^{-} \times C_{\text {load }} / \mathrm{pF} \\
& \Rightarrow \mathrm{S} / \mathrm{N} \approx 13 \text { for } 22 \mathrm{~cm} \text { long ladder }
\end{aligned}
$$

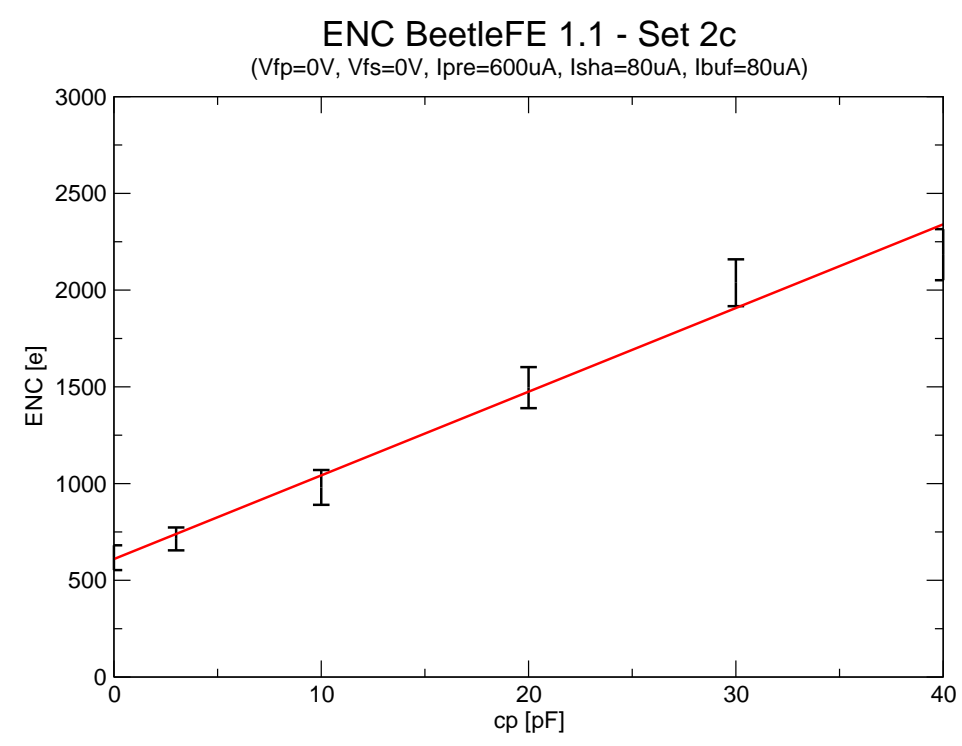




\section{Read-Out Hybrid}

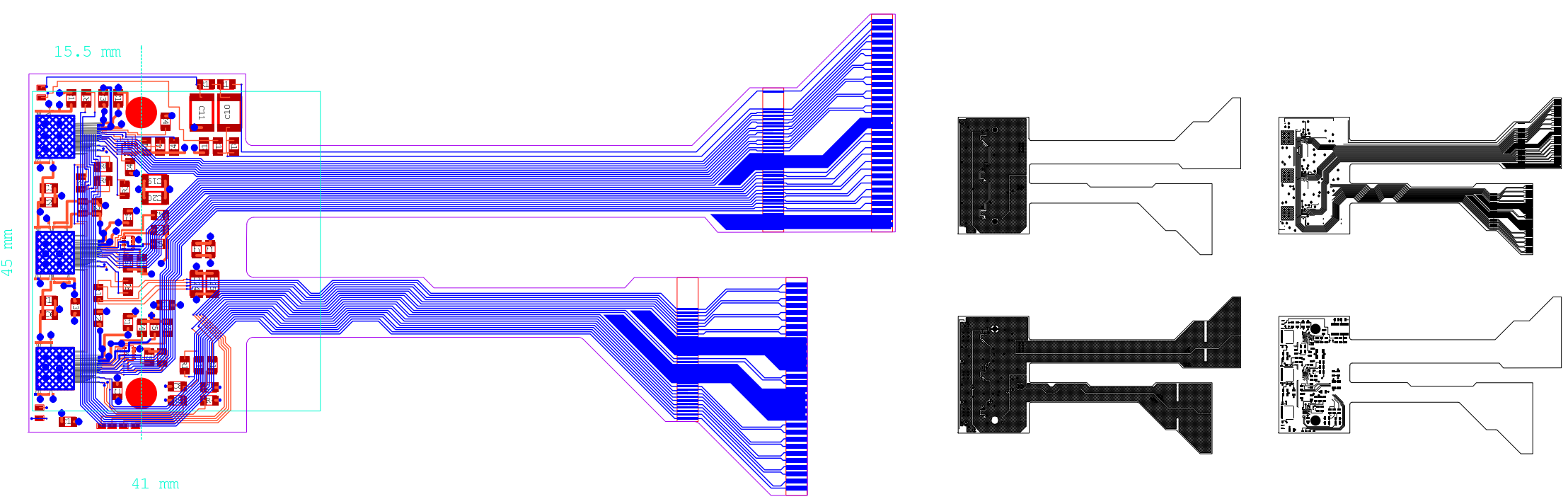

Four-layer kapton hybrid for three Beetle chips

\section{1. digital/analog power \\ 3. traces \\ 2. digital/analog ground \\ 4. components}

Two-layer "tails" for control signals and read-out of analog signals Separate pitch adaptor ( thin-film ceramic?)

$$
\Rightarrow \text { prototypes in production }
$$




\section{Read-Out Link}

Readout rate (L0-trigger accept rate): $1.1 \mathrm{MHz}$

$$
\Rightarrow \text { Beetle output: } 4 \times 40 \mathrm{MHz} \text { analog }
$$

8-bit digitization:

$$
\Rightarrow 1.28 \mathrm{Gbit} / \mathrm{s} \text { per Beetle, } 3.84 \mathrm{Gbit} / \mathrm{s} \text { per ladder, } 134 \mathrm{Gbit} / \mathrm{s} \text { per Box }
$$

Design criteria read-out link:

radiation hardness components, material budget, cost

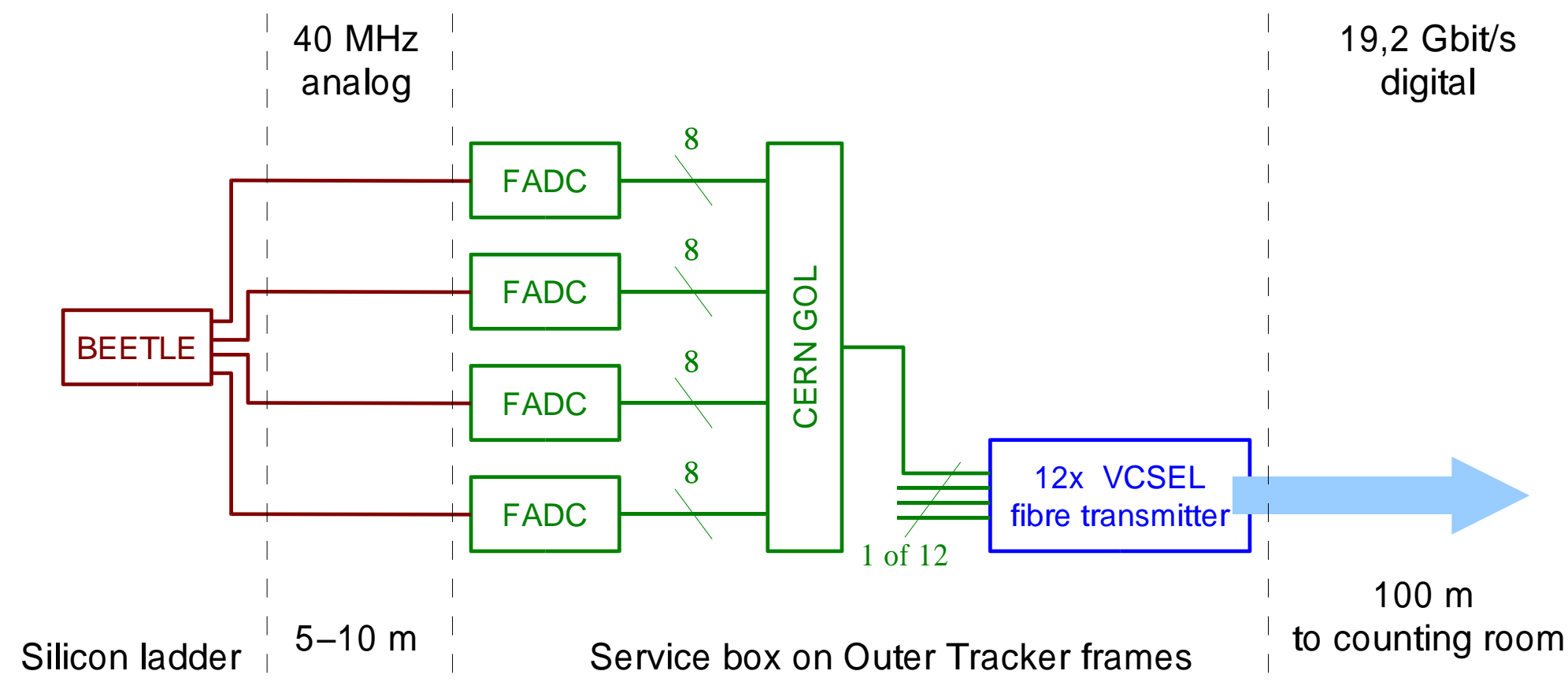




\section{"LHCb-Light"}
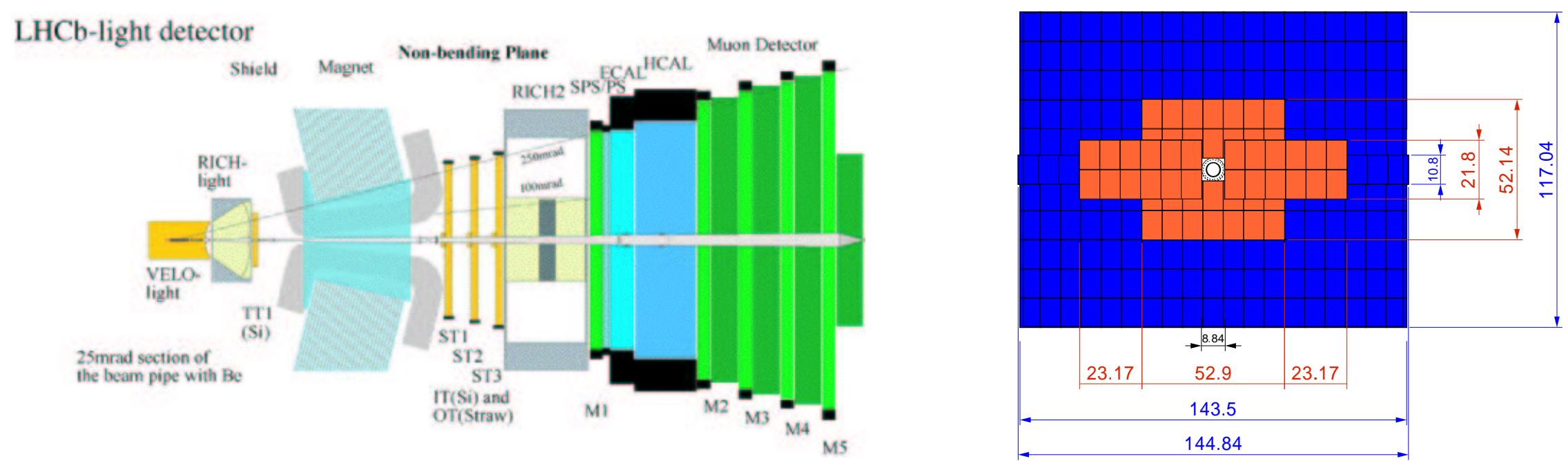

Common effort for overall re-optimization of $\mathrm{LHCb}$ detector Two major changes to tracking system under investigation:

a) reduce number of tracking stations

- improved performance due to lower material budget

- baseline scenario: four stations

$\Rightarrow$ very promising results b) use $1^{\text {st }}$ station in Level-1 trigger

- improved performance from momentum information

- may need all silicon $\left(6.8 \mathrm{~m}^{2}\right)$

$\Rightarrow$ careful study still needed 


\section{Summary and Outlook}

\section{Main requirements:}

- $22 \mathrm{~cm}$ long ladders,

- large pitch ( $80 \mu \mathrm{m}$ resolution sufficient)

- fast shaping for $25 \mathrm{~s}$ bunch crossing rate

- material budget!!!

\section{Main R\&D:}

- sensors: 6" $\mathrm{p}^{+}$-on-n, $320 \mu \mathrm{m}$ thick, $198 \mu \mathrm{m}$ or $237.5 \mu \mathrm{m}$ pitch

- readout chip: Beetle

- ladder supports, detector boxes: modular design, low-weight materials

- digital optical read-out link

\section{Upcoming:}

- May/June — testbeam with full-size prototype sensors

$\Rightarrow$ validation of sensor design, choice of strip geometry

- October - submission Technical Design Report 